\title{
Effects of Fluorodeoxyglucose Conjugated and Unconjugated Iron Oxide Magnetic Nanoparticles on Macrophages: a Pilot Study
}

Jenson Lim ${ }^{1}$

https://orcid.org/0000-0001-7417-356X

Michaela Petaroudi ${ }^{2}$

https://orcid.org/0000-0002-1982-913X

Gillian Pearce ${ }^{3}$

https://orcid.org/ 0000-0003-1428-2021

Volkan Yasakçi ${ }^{4}$

https://orcid.org/0000-0002-4133-3886

Perihan Unak ${ }^{4^{*}}$

https://orcid.org/0000-0002-5464-2987

${ }^{1}$ University of Stirling, Biological and Environmental Sciences, Faculty of Natural Sciences, Stirling, UK; ${ }^{2}$ University of Glasgow, Centre for the Cellular Microenvironment, Glasgow, UK; ${ }^{3}$ Aston University, School Of Engineering and Applied Sciences, Birmingham, UK; ${ }^{4}$ Ege University, Institute of Nuclear Sciences, Department of Nuclear Applications, Bornova, Izmir, Turkey

Editor-in-Chief: Alexandre Rasi Aoki

Associate Editor: Daniel Fernandes

Received: 2020.11.21; Accepted: 2021.04.12.

*Correspondence: perihan.unak@gmail.com; Tel.: +90-536-5131806 (P.U.).

\section{HIGHLIGHTS}

- Iron oxide based nanoparticles were synthesized.

- They can be linked to macrophages without the function of the macrophages being affected.

- No detrimental effects to the macrophages were evident.

Abstract: The effects of fluorodeoxyglucose conjugated iron oxide magnetic nanoparticles (FDGMNP) on macrophages are presented using a yeast substrate. Iron oxide magnetic nanoparticles (MNP) were synthesized by partially reducing $\mathrm{FeCl}_{3}$, then conjugated with (3-aminopropyl) triethoxysilane (APTES) after silication with tetraethyl orthosilicate. Silanated MMP nanoparticles were combined with fluorodeoxyglucose (FDG). Fluorodeoxyglucose iron oxide magnetic nanoparticles (FDGMNP) and its unconjugated control (MNP) were added $(1 \mathrm{~mL})$ to the cells from the murine macrophage-like, Abelson murine leukemia virus transformed cell line RAW 264.7 (American Type Culture Collection number TIB-71) cell culture wells at different concentrations from $90-3.6 \mu \mathrm{g} / \mathrm{mL}$. Cells were placed on the magnet plate for 30 min before incubating at $37^{\circ} \mathrm{C}, 5 \% \mathrm{CO}_{2}$ overnight. MTT (3-(4,5-dimethylthiazol-2-yl)-2,5-diphenyltetrazolium bromide tetrazolium) assay was performed to measure cell viability. Our results demonstrate that iron based nanoparticles can be linked to macrophages (elements of the immune system that attack bacteria) without 
the function of the macrophages being affected, ie no detrimental effects to the macrophages were evident in these experiments. We conclude that neither FDGMNP nor MNP had a detrimental effect on macrophage function.

Keywords: urinary tract infections; iron oxide nanoparticles; fluorodeoxyglucose; magnetic nanoparticles.

\section{INTRODUCTION}

In the European Union (EU) alone, it is believed that 25000 patients die (in the EU alone each year) on account of bacterial infections acquired in hospitals. Infections from resistant bacteria, can be life-threatening for example, with pneumonia, wound infections and sepsis [1]. In addition to causing deaths and extensive suffering, antimicrobial resistance potentially has large scale economic impacts. Multidrug-resistant bacteria are estimated to result in an economic loss in excess of $€ 1.5$ billion alone each year in the EU. Such infections constitute an on-going problem in hospitals and other such environments, and not only give rise to lifethreatening infections such as sepsis, but also cause problems at other more local sites in the body. [1]

In addition to problems arising in hospitals and other such heath care settings, bacterial resistance is also becoming a problem in the treatment of more common infections in the community eg urinary tract infections (UTI's). In most cases the causative agent of a UTI ( $75 \%$ of UTI cases) is Escherichia coli, (E. coli) [2]. UTIs occur more frequently in females, and it is believed that approximately half of all females contact one or more UTIs during their lifespan [3]. It is well recognised that bacteria causing UTIs embed themselves in a biofilm in the bladder [4]. The biofilm, (about 50 microns thick) protects the bacteria and provides nutrients to the bacteria [5]. Bacterial resistance arises on account of mechanisms which include the production of enzymes that inactivate antibiotics (e.g. $\beta$-lactamases), and biofilm growth [6,7]. Even when alternative antibiotics are developed, bacteria still have the potential to become resistant to such novel antibiotics [6].

Clearly, there is scope for new treatments and new approaches to combat microbial resistance in order to relieve the suffering caused by such infections. Such new treatments have involved the use of nanoparticles composed of eg iron oxide, and chitosan nanoparticles. [8]. For example, Ibrahim et al (2015) investigated the use of chitosan nanoparticles with antibiotics such as ciprofloxacin, chlortetracycline hydrochloride and gentamycin sulphate and found that the use of this nanoparticle-antibiotic chimera inhibited growth of gram (+) and gram (-) bacteria. Furthermore, Jena and coauthors [9] demonstrated that CS-AgNPs inhibited bacterial growth without significant damage to macrophages. Furthermore it was demonstrated that propidium iodide staining showed endocytosis of CS-AgNPs resulting in reduced intracellular bacterial survival in macrophage resistance [9]. Chitosan nanoparticles in particular have been shown to be particularly effective in potential UTI treatment. Lambert and coauthors demonstrated that chitosan nanoparticles could effectively be taken up by pathogenic bacteria such as $E$. coli and $S$. aureus. [10]. So there is a clear role for metal based nanoparticles in the killing of pathogenic bacteria [10]. Metal-based nanoparticles are the most popular inorganic nanoparticles and represent a promising solution against the resistance to traditional antibiotics. Not only do they use mechanisms of action that are completely different from those described for traditional antibiotics, exhibiting activity against bacteria that have already developed resistance, but they also target multiple biomolecules compromising the development of resistant strains [10].

Iron oxide magnetic nanoparticles have potential for imaging, diagnosis, treatment, and separation of biological materials. These nanoparticles can be oriented in the magnetic field due to their magnetic properties. Due to its magnetic properties, it is also possible to direct drug molecules loaded on magnetic nanoparticles to localized disease sites in the body. The aim of this study is to examine the use of fluorodeoxyglucose conjugated MNPs in imaging and therapy of infection, both as an MRI (Magnetic Resonance Imaging) agent, and for imaging of infection and when the drug molecule is loaded. In this way, higher drug concentrations can be emitted through the magnetic field for an infection in an area where the antibiotic is not easily accessible.

The aim of this work is to understand if FDG MNPs can be linked to macrophages without the function of the macrophages being affected detrimentally. Thus, it may be possible to imaging infection disease using these particles such as MRI imaging systems. 


\section{MATERIALS AND METHODS}

\section{Reagents and equipments}

$\mathrm{FeCl}_{3}$, tetraethyl orthosilicate (TEOS), (3-aminopropyl) triethoxysilane, cysteamine, $\mathrm{C} 18$ pre-cartridge, Dowex 50 cation exchange resin column, were purchased from Sigma-Aldrich Co. (Darmstadt, Germany). Dimethylformamide, sodium fluoride, sodium sulphite, Kryptofix, N-hydroxysuccinimide, ammonia solution were obtained from Merck Co. (Darmstadt, Germany). Mannose triflate, Potassium carbonate, Ambersep 900 quaternary ammonium anion exchange resin were supplied from Fluka Co. (Buchs, Switzerland). All other chemicals were supplied from Sigma-Aldrich Co. (Darmstadt, Germany). All of the solvents and reagents were of the highest purity and were used as supplied.

The following equipment from Ege University Institute of Nuclear Sciences were used: The dynamic light scattering device (Malvern Nano ZS DLS, Malvern, UK). Inductively Coupled Plasma and Mass Spectrometry (ICP-MS) (Agilent Technologies 7900 ICP-MS SPS4 Auto sampler, California, USA). Scanning electron microscopy (SEM) (FEI QUANTA FEG 250, Thermo Fisher Scientific, Oregon, USA) images were taken at Tekirdağ Namik Kemal University, NABILTEM, Turkey.

\section{Synthesis Procedure}

Synthesis of mNPs was undertaken as described in previous reports [11,17]. Briefly, $2 \mathrm{M} \mathrm{FeCl}_{3}$ was combined with $80 \mathrm{mM} \mathrm{Na}_{2} \mathrm{SO}_{3}$ prior to the addition of $25 \% \mathrm{NH}_{3}$ solution under inert medium. The particles were separated by centrifugation and then washed with a water-ethanol (2:1) mixture and re-suspended in $80 \%$ ethanol after 30 minutes of incubation at $70{ }^{\circ} \mathrm{C}$. Then they were mixed with tetraethyl orthosilicate for 12 hours at $40^{\circ} \mathrm{C}$ and washed with methanol prior to incubation with (3-aminopropyl) triethoxysilane (APTES) for 12 hours at $60^{\circ} \mathrm{C}$ with rapid stirring.

Separately, to synthesize FDG-mNPs, solutions of mannose triflate and cysteamine were prepared in water, mixed and heated for 1 hour at $90{ }^{\circ} \mathrm{C}$, precipitated and dried overnight prior to dissolving in dimethylformamide. Next, solutions of Kryptofix, $\mathrm{K}_{2} \mathrm{CO}_{3}$, dimethylformamide and $\mathrm{NaF}$ were added to $1 \mathrm{~mL}$ of the prepared mannose triflate-cysteamine and heated for 20 minutes at $90{ }^{\circ} \mathrm{C}$. The product was purified by sequential passing through a Dowex 50 cation exchange resin column, Ambersep 900 quaternary ammonium anion exchange resin, Amberlite anion exchange resin and finally a $\mathrm{C} 18$ pre-cartridge. The purified $\mathrm{NaF}$ substituted mannose triflate-cysteamine was mixed with the MNPs prior to the addition of $\mathrm{N}$-hydroxysuccinimide and stirred for 2 hours.

\section{Characterization of mNPs and FDG-mNPs}

The hydrodynamic diameter of the FDG-mNPs was measured using a dynamic light scattering device. For that the MNPs or FDGMNP $\mathrm{s}$ were dispersed in 20\% dextrose solution at FDGMNP $\left(100 \mu \mathrm{g} \mathrm{mL}^{-1}\right)$ concentration and measured at $25{ }^{\circ} \mathrm{C}$. Measurements were repeated three times, and the results were expressed as the mean \pm standard deviation. Particle size and morphology of the synthesized MNPs were obtained using scanning electron microscopy (SEM). Iron concentration was measured using an Inductively Coupled Plasma and Mass Spectrometry (ICP-MS, Agilent Technologies 7900 ICP-MS SPS4 Auto sampler, California, USA).

The synthesized MNP and FDGMNP were maintained at $4{ }^{\circ} \mathrm{C}$ at a density of $30 \mathrm{mg} / \mathrm{ml}$ in solution. RAW 264.7 (TIB-7) cells were acquired from ATCC (American Type Culture Collection). The magnetic plate used to generate the magnetic field for magnetofection was from Ozbioscience (Marseilles, France). Heat-Killed (HK) Candida albicans SC5314 was gifted by from Rebecca Hall (Kent) and was maintained in the fridge. All other chemical reagents were purchased from Sigma-Aldrich or Fisher Scientific.

\section{Macrophage cell growth conditions}

Cells from the murine macrophage-like, Abelson murine leukemia virus transformed cell line RAW 264.7 (American Type Culture Collection number TIB-71) were cultured in DMEM supplemented with $2 \mathrm{mM} \mathrm{L-}$ glutamine and $10 \%$ heat-inactivated $\mathrm{FBS}$ at $37^{\circ} \mathrm{C}, 5 \% \mathrm{CO}_{2}$. As required, macrophages were scrapped in PBS, counted, and seeded in complete medium (500,000/well) onto standard clear 24-well tissue culture plates and incubated for $24 \mathrm{~h}$ at $37^{\circ} \mathrm{C}, 5 \% \mathrm{CO}_{2}$. Cells were serum starved for $2-16$ hours prior to experimental use. 


\section{Nanoparticle addition and cell viability}

Fluorodeoxyglucose iron oxide magnetic nanoparticles (FDGMNP) and its unconjugated control (MNP) were added $(1 \mathrm{~mL})$ to the wells at different concentrations from $90-3.6 \mu \mathrm{g} / \mathrm{mL}$. Cells were placed on the magnet plate for 30 min before incubating at $37^{\circ} \mathrm{C}, 5 \% \mathrm{CO}_{2}$ overnight. MTT assay was performed to measure cell viability [12]. Briefly cells were washed in PBS before $0.5 \mathrm{mg} / \mathrm{ml}$ of the water-soluble tetrazolium dye MTT (3-(4,5-dimethylthiazol-2-yl)-2,5-diphenyltetrazolium bromide, prepared in SFM) was added to wells. For negative control, $0.1 \%(\mathrm{v} / \mathrm{v})$ of the detergent Triton X-100 was introduced to a well containing cells and incubated at room temperature for $5 \mathrm{~min}$, washed in PBS and assayed with MTT. After addition of MTT, a 24-well plate was incubated at $37^{\circ} \mathrm{C}$ and $4 \% \mathrm{CO}_{2}$ for $2 \mathrm{hr} 30 \mathrm{~min}$. Following incubation, the MTT mixture was removed and any purple formazan formed was dissolved in DMSO. Absorbance at $595 \mathrm{~nm}$ was measured using a plate reader (Molecular Devices) and the Software Pro 5.4 software.

\section{Statistical analyses}

One-way ANOVA followed by Dunnett's multiple comparisons test was performed using GraphPad Prism version 8.4.3 for Windows, GraphPad Software, San Diego, California USA, www.graphpad.com.

\section{RESULTS}

The nanoparticles were homogeneously dispersed in dextrose (80\% water, $20 \%$ dextrose) solution according to DLS measurement. The medium size was $176.7 \pm 4.7 \mathrm{~nm}(\mathrm{n}=5)$ together with their hydrodynamic radius similar to previous study [11]. The surface potential of MNPs and FDGMNPs were found to be -4.77 $\pm 0.918 \mathrm{mV}$ and $21.26 \pm 0.862 \mathrm{mV}$ respectively.

SEM and TEM images showed that FDGMNPs displayed a mean size of 10-20 nm. Additionally, SEM data showed that these FDGMNPs had a cubic spinel structure (Figure 1A, Figure1B). MNPs possessed a particle shape that was a uniform homogeneous cubic crystal structure, with a particle size of around 20-30 nm (Figure 1B).

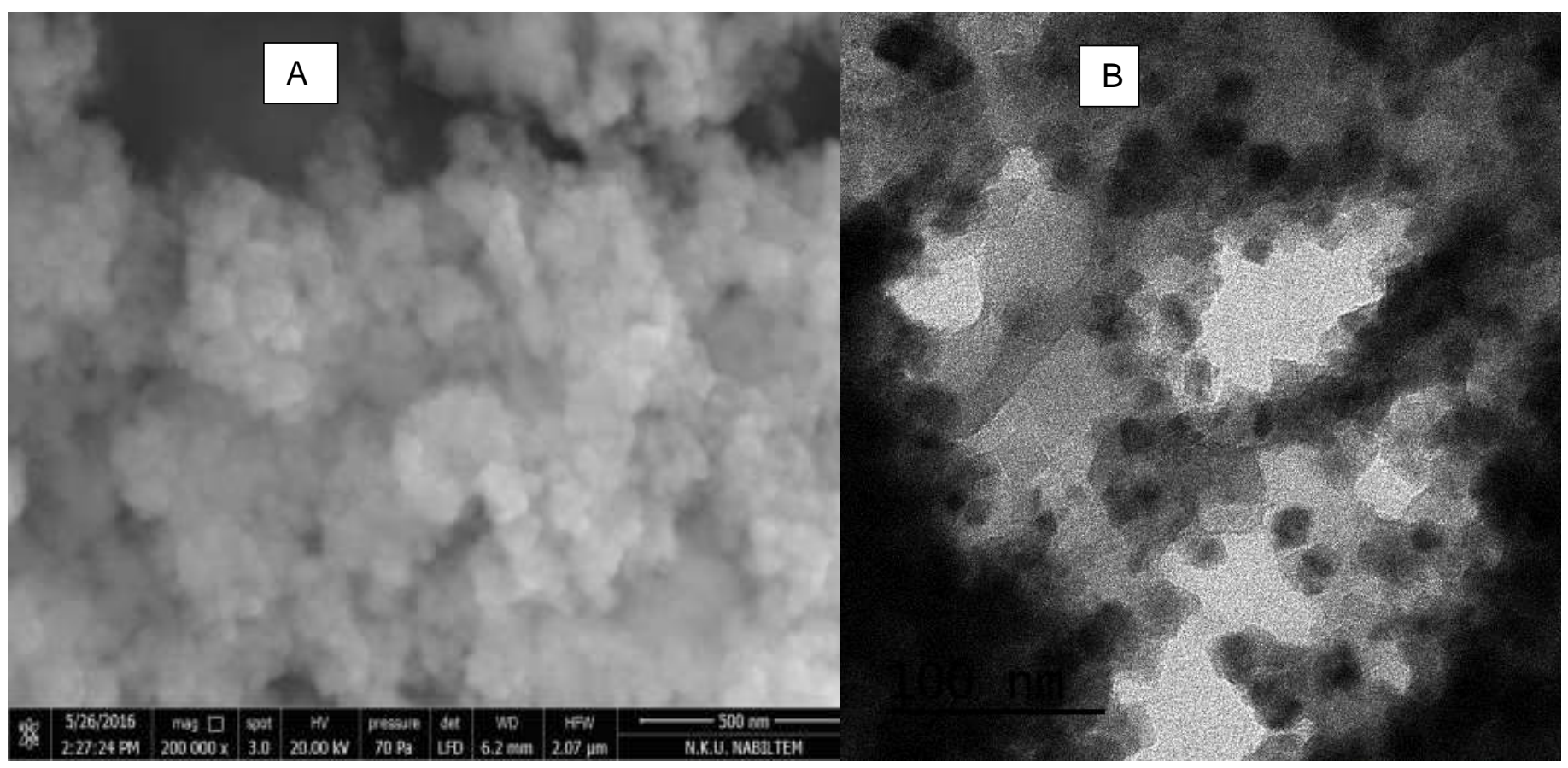

Figure 1. A) SEM Image of FDGMNPs (Fluorodeoxyglucose iron oxide magnetic nanoparticles), B) TEM Image of FDGMNPs (Fluorodeoxyglucose iron oxide magnetic nanoparticles) 
Iron oxide based magnetic particles with and without conjugation with fluorodeoxyglucose (FDGMNP and MNP respectively)) successfully link to macrophages. The macrophages attached to the MNP and FDGMNP were then allowed to act on yeast (readily available and safe to use). The results is shown in Figure 2.

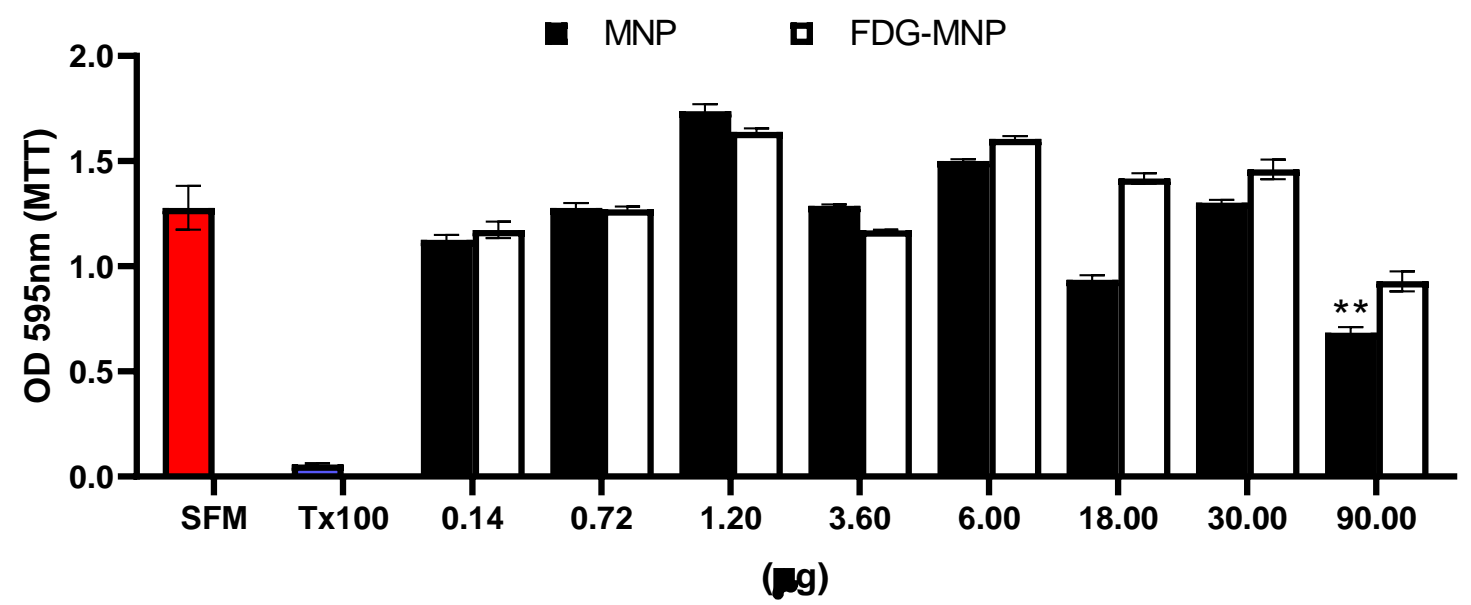

Figure 2. The effects of FDGMNP (Fluorodeoxyglucose iron oxide magnetic nanoparticles) linked to macrophages and MNP (Iron oxide magnetic nanoparticles) linked to macrophages on yeast.

FDGMNP has little effect on RAW 2647 macrophage viability (Figure 2). Serum starved RAW264.7 murine macrophages were challenged with FDG-MNP, MNP, Triton X-100 or untreated (serum-free medium, SFM) for $30 \mathrm{~min}$ at $37^{\circ} \mathrm{C}$. Cell viability was determined by MTT assay and no detrimental effects to the macrophages was observed. Statistical significance compared to SFM was determined by two-way ANOVA and a Tukey's multiple comparisons test. $\left({ }^{* *}\right) p \leq 0.01$.

\section{DISCUSSION}

Magnetic nanoparticles (MNP) have potential in medical sciences, for example; PET-MRI (Positron Emission Tomography-Magnetic Resonance Imaging) as contrast agent and cell labelling [12]. Iron oxide MNPs have the potential to be used in two ways, either as targeted therapy agents to target chemotherapy drugs for cancer therapy or as magnetic fluid hyperthermia. The first applications of MNPs in vivo were done in 2011 by Nacev and coauthors, who investigated the possibility of delivering drug chemotherapy through MNPs linked to chemotherapeutic agents [13]. The goal involved in this treatment was achieved using an external magnetic field applied around the area of cancerous tissue. This area resulted in MNPs collected in cancer cells providing a high concentration of MNP and a higher drug concentration in cancer cells. Another study involving the therapeutic uses of MNPs was conducted in 2013 by Huang and Hainfeld, who conducted two studies using iron oxide MNPs [14]. The first study involved MNPs attached to liposomes containing a chemotherapeutic drug called doxorubicin. In the second study, MNPs were covalently bound to doxorubicin coated in a thermosensitive polymer. Both techniques took advantage of the heat generated in the magnetic field applied by MNPs to break the bonds that release the chemotherapeutic drug to destroy cancerous cells. With this application, possible damage to the healthy tissues surrounding the cancer has been greatly reduced.

Increased metabolic activity of tumor cells is induced by overexpression of glucose transporters within the cell membrane. This leads to an increase in glucose uptake. There are different types of glucose transporters, but one seems to be particularly important, namely the GLUT-1 receptor expressed in all cancerous cells. Visualization of GLUT-1 receptors in tumor cells is possible with MRI scanning using superparamagnetic iron oxide MNPs as contrast agents [15] .However, in order for MNPs to be taken up by GLUT-1 receptors for display, they are functionalized with a glucose analog, namely 2-deoxy-D-glucose (2DG). This analog increases the uptake of MNPs in cancer cells due to the Warburg effect (higher metabolic uptake of the FDG component of the FDGMNP hybrid). It has been shown that FDGMNP have high affinity for MCF-7 breast cancer cells according to previous studies [11]. In a study conducted with another group, FDGMNPs were injected both intratumorally and intravenously (via the tail vein) in in-vivo studies on intraabdominal tumors of mice [16]. Mice were euthanized 70 minutes to 12 hours after injection. All of the mice showed little pronounced uptake of FDGMNPs into tumor cells in cells of other organs, in the heavy and 
histology of cancer tissue. In addition, the results of in-vivo experiments in mice with prostate cancer over several days also showed that FDGMNPs were more concentrated in cancer cells of the prostate [17]. Thus, such nanoparticles show promise in the treatment of cancer hyperthermia. Clearly nanoparticles have a plethora of applications in cancer treatment as well as potential uses in treating bacterial infections including those bacteria involved in causing urinary tract infections. It is also known that Candida (yeast species) can also cause UTI's, and results of our study (using a yeast species) has shown that FDGMNPs and MNPs do not affect the functionality of macrophages, which are involved in the immune response to such infections. We believe therefore that FDGMNPs and MNPs have potential uses in the treatment of UTI's caused by yeast and bacterial species. [18]

FDG MNPs can be linked to macrophages (elements of the immune system that attack bacteria) without the function of the macrophages being affected, ie no detrimental effects to the macrophages were evident in these experiments. FDG is known to be taken up by bacteria [19]. ${ }^{18} \mathrm{~F}-\mathrm{FDG}$ is one of the few tracers that can be clinically applied to imaging infection. In this study, higher uptake was seen with increasing FDG MNP concentrations. FDG also appears to increase iron retention at increasing concentrations.

Other reports have also indicated that nanoparticles do not appear to have a detrimental effect on macrophages [9], and that the effects of antibiotics can be enhanced by the use of nanoparticles [8]. Furthermore Lambert and coauthors demonstrated a high affinity of chitosan for S.Aureus and E.Coli [10]. As a result MNPs seems to be very promising that has shown potential application in targeting different strains of resistant and nonresistant microbial strains is the treatment with FDG MNPs.

To date, ${ }^{18} \mathrm{~F}$-FDG is one of the few tracers that may be clinically applied in infection imaging FDG MNPs can be taken up by a wide range of commonly encountered bacterial pathogens. Bacterial pathogens may also take up FDG MNP in vivo, thereby contributing to the MRI signal of iron oxide in infection imaging. Future experiments are needed to determine the proportion of FDG MNP that is taken up by infecting bacteria as compared to the ${ }^{18} \mathrm{~F}$-FDG taken up by inflammatory cells. This will unveil the significance of our present findings in the clinical context.

\section{CONCLUSION}

Iron based MNPs can be linked to macrophages without the function of the macrophages being affected detrimentally. FDG increased the MNP uptake to macrophages. Although our study is based on yeast, this approach may have applications to other microbes and in particular those causative agents involving UTI's.

Macrophages linked to chitosan and perhaps other nanoparticles could be used as a potential treatment for UTI's. Such treatments could possibly include the introduction of macrophages linked to nanoparticles directly into the biofilm itself, and this will be the focus of future investigation.

Funding: This research received no external funding.

Conflict of interest: The authors declare that they have no conflict of interest.

\section{REFERENCES}

1. Prestinaci F, Pezzotti P, Pantosti A. Antimicrobial resistance: a global multifaceted phenomenon. Pathog Glob Health. 2015;109(7):309-18.

2. Flores-Mireles AL, Walker JN, Caparon M, Hultgren SJ. Urinary tract infections: epidemiology, mechanisms of infection and treatment options. Nat Rev Microbiol. 2015 May;13(5):269-84.

3. Aydin A, Ahmed K, Zaman I, Khan MS, Dasgupta P. Recurrent urinary tract infections in women. Int Urogynecol J. 2015 Jun;26(6):795-804.

4. Tenke P, Köves B, Nagy K, Hultgren SJ, Mendling W, Wullt B, Grabe M, Wagenlehner FM, Cek M, Pickard R, Botto $\mathrm{H}$, Naber KG, Bjerklund Johansen TE. Update on biofilm infections in the urinary tract. World J Urol. 2012 Feb;30(1):51-7.

5. Khatoon Z, McTiernan CD, Suuronen EJ, Mah TF, Alarcon El. Bacterial biofilm formation on implantable devices and approaches to its treatment and prevention. Heliyon. 2018 Dec 28;4(12):e01067.

6. Kahlmeter G, Åhman J, Matuschek E. Antimicrobial Resistance of Escherichia coli Causing Uncomplicated Urinary Tract Infections: A European Update for 2014 and Comparison with 2000 and 2008. Infect Dis Ther. 2015 Dec;4(4):417-23.

7. Cormode DP, Gao L, Koo H. Emerging Biomedical Applications of Enzyme-Like Catalytic Nanomaterials. Trends Biotechnol. 2018 Jan;36(1):15-29.

8. Ibrahim HM, El-Bisi MK, Taha GM, El-Alfy EA. Chitosan nanoparticles loaded antibiotics as Drug Delivery bio material. J App Pharm Sci, 2015; 5 (10): 85-90. 
9. Jena $P$, Mohanty S, Mallick R, Jacob B, Sonawane A. Toxicity and antibacterial assessment of chitosan-coated silver nanoparticles on human pathogens and macrophage cells. Int J Nanomedicine. 2012;7:1805-18.

10. Lambert T, Santiesteban R, Ceiro WG, Fernandez ME, Lopez GD, Corrales WC, Effect of bioproducts in the production of Phaseolus vulgaris L. and Arachis hipogea L., Rev De Cienc Agric, 2019;36(1)59-66.

11. Ozkaya F, Unak P, Medine EI, Sakarya S, Unak G, Timur S. 18FDG conjugated magnetic nanoparticle probes: synthesis and in vitro investigations on MCF-7 breast cancer cells. J Radioanal Nucl Chem. 2013;295:1789-96.

12. Caron E, Hall A. Identification of two distinct mechanisms of phagocytosis controlled by different Rho GTPases. Science. 1998 Nov 27;282(5394):1717-21.

13. Nacev A, Kim SH, Rodriguez-Canales J, Tangrea MA, Shapiro B, Emmert-Buck MR. A dynamic magnetic shift method to increase nanoparticle concentration in cancer metastases: a feasibility study using simulations on autopsy specimens. Int J Nanomedicine. 2011;6:2907-23.

14. Huang HS, Hainfeld JF. Intravenous magnetic nanoparticle cancer hyperthermia. Int J Nanomedicine. 2013;8:252132.

15. Shan XH, Hu H, Xiong F, Gu N, Geng XD, Zhu W, et al. Targeting Glut1-overexpressing MDA-MB-231 cells with 2deoxy-D-g1ucose modified SPIOs. Eur J Radiol. 2012 Jan;81(1):95-9.

16. Watkins AJ, Pearce G, Unak P, Guldu OK, Yasakci V, Akin O, et al. Tissue Morphology and Gene Expression Characterisation of Transplantable Adenocarcinoma Bearing Mice Exposed to Fluorodeoxyglucose-Conjugated Magnetic Nanoparticles. J Biomed Nanotechnol. 2018 Nov 1;14(11):1979-91.

17. Aras O, Pearce G, Watkins AJ, Nurili F, Medine El, Guldu OK, T et al. An in-vivo pilot study into the effects of FDGmNP in cancer in mice. PLoS ONE 2018; 13(8): e0202482.

18. Dinali R, Ebrahiminezhad A, Manley-Harris M, Ghasemi $Y$, Berenjian A. Iron oxide nanoparticles in modern microbiology and biotechnology. Crit Rev Microbiol. 2017 Aug;43(4):493-507.

19. Heuker M, Sijbesma JWA, Aguilar Suárez R, De Jong JR., Boersma HH, Luurtsema G. et al. In vitro imaging of bacteria using ${ }^{18} \mathrm{~F}$-fluorodeoxyglucose micro positron emission tomography Sci Rep. 2017;7(1):4973.

(C) $(5)$
EY 2021 by the authors. Submitted for possible open access publication under the terms and
conditions of the Creative Commons Attribution (CC BY NC) license
(https://creativecommons.org/licenses/by-nc/4.0/). 